\title{
Balancing near-field enhancement, absorption, and scattering for effective antenna-reactor plasmonic photocatalysis
}

Kun Li, Nathaniel J. Hogan, Matthew Kale, Naomi J. Halas, Peter Nordlander, and Phillip Christopher

Nano Lett., Just Accepted Manuscript • Publication Date (Web): 08 May 2017

Downloaded from http://pubs.acs.org on May 9, 2017

\section{Just Accepted}

"Just Accepted" manuscripts have been peer-reviewed and accepted for publication. They are posted online prior to technical editing, formatting for publication and author proofing. The American Chemical Society provides "Just Accepted" as a free service to the research community to expedite the dissemination of scientific material as soon as possible after acceptance. "Just Accepted" manuscripts appear in full in PDF format accompanied by an HTML abstract. "Just Accepted" manuscripts have been fully peer reviewed, but should not be considered the official version of record. They are accessible to all readers and citable by the Digital Object Identifier (DOI®). "Just Accepted" is an optional service offered to authors. Therefore, the "Just Accepted" Web site may not include all articles that will be published in the journal. After a manuscript is technically edited and formatted, it will be removed from the "Just Accepted" Web site and published as an ASAP article. Note that technical editing may introduce minor changes to the manuscript text and/or graphics which could affect content, and all legal disclaimers and ethical guidelines that apply to the journal pertain. ACS cannot be held responsible for errors or consequences arising from the use of information contained in these "Just Accepted" manuscripts. 


\title{
Balancing near-field enhancement, absorption, and scattering for effective antenna-reactor plasmonic photocatalysis
}

\author{
Kun Li ${ }^{1}$, Nathaniel J. Hogan ${ }^{2,3}$, Matthew J. Kale ${ }^{1,4}$, Naomi J. Halas 2,3,5,6, Peter Nordlander 2,3,6, \\ Phillip Christopher ${ }^{*}, 1,7,8$
}

1. Department of Chemical and Environmental Engineering, University of California, Riverside, Riverside, CA 92521, USA; 2. Department of Physics and Astronomy, Rice University, Houston, TX 77005, USA; 3. Laboratory for Nanophotonics, Rice University Houston, TX 77005, USA; 4. Division of Chemistry and Chemical Engineering, California Institute of Technology, Pasadena, CA 91125, USA; 5. Department of Chemistry, Rice University, Houston, TX 77005, USA; 6. Department of Electrical and Computer Engineering, Rice University, Houston, TX 77005, USA; 7. Program in Materials Science, University of California, Riverside, Riverside, CA 92521, USA; 8. UCR Center for Catalysis, University of California, Riverside, Riverside, CA 92521, USA

* Corresponding Author

Email: christopher@engr.ucr.edu 


\begin{abstract}
\end{abstract}
Efficient photocatalysis requires multifunctional materials that absorb photons and generate energetic charge carriers at catalytic active sites to facilitate a desired chemical reaction. Antenna-reactor complexes are an emerging multifunctional photocatalytic structure where the strong, localized near field of the plasmonic metal nanoparticle (e.g. Ag) is coupled to the catalytic properties of the non-plasmonic metal nanoparticle (e.g. Pt) to enable chemical transformations. With an eye towards sustainable solar driven photocatalysis we investigate how the structure of antenna-reactor complexes governs their photocatalytic activity in the lightlimited regime, where all photons need to be effectively utilized. By synthesizing core@shell/satellite $\left(\mathrm{Ag} @ \mathrm{SiO}_{2} / \mathrm{Pt}\right)$ antenna-reactor complexes with varying $\mathrm{Ag}$ nanoparticle diameters and performing photocatalytic $\mathrm{CO}$ oxidation, we observed plasmon-enhanced photocatalysis only for antenna-reactor complexes with antenna components of intermediate size (25 and $50 \mathrm{~nm}$ ). Optimal photocatalytic performance was shown to be determined by a balance between maximized local field enhancements at the catalytically active Pt surface, minimized collective scattering of photons out of the catalyst bed by the complexes, and minimal light absorption in the Ag nanoparticle antenna. These results elucidate the critical aspects of local field enhancement, light scattering, and absorption in plasmonic photocatalyst design, especially under light-limited illumination conditions. 


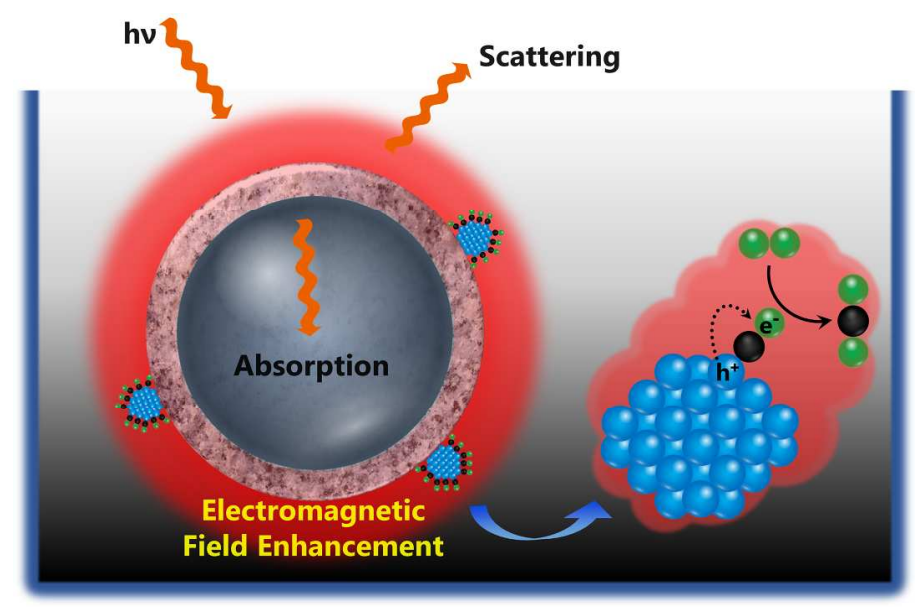

KEYWORDS: Localized surface plasmons, electromagnetic field enhancement, antennareactor, photocatalysis, size dependence, light-limited

\section{MAIN TEXT}

The utilization of solar energy to drive photocatalysis has been demonstrated for a wide range of important reactions, offering approaches for sustainable fuel production, ${ }^{1}$ pollutant remediation, ${ }^{2}$ and structurally complex organic compound synthesis. ${ }^{3}$ While the design of heterogeneous photocatalysts has focused largely on semiconducting light absorbers, ${ }^{4,5}$ it has recently been demonstrated that metallic nanostructures offer unique characteristics for facilitating photocatalytic reactions. ${ }^{6-9}$ Strong light absorption through the excitation of localized surface plasmons on plasmonic metal nanostructures ${ }^{10}$ comprised of $\mathrm{Au},{ }^{11-13} \mathrm{Ag},{ }^{14-16} \mathrm{Cu},{ }^{17}$ or $\mathrm{Al},{ }^{18}$ has been shown to drive photocatalytic processes through the excitation and transient transfer of energetic, or hot, carriers to adsorbates. ${ }^{19-24}$ However, there is limited evidence that the energies of hot carriers in plasmonic nanostructures can be selectively tailored to target specific catalytic reaction pathways, ${ }^{25,26}$ and plasmonic nanostructures are optimal catalysts for only a very few 
relevant industrial chemical processes. It has also been shown that irradiation of non-plasmonic metal nanostructures (e.g. Pt, Pd, Rh, Ru), a category that comprises the vast majority of commercial supported metal catalysts, by relatively low-intensity visible light, can increase the rate of catalyzed reactions. ${ }^{27-30}$ Illumination of non-plasmonic metal nanostructures can also enable control of reaction selectivity through resonant photoexcitation of bond-specific adsorbate-metal electronic transitions. ${ }^{31}$ Despite their promise of light-controlled reaction specificity, the lack of a viable mechanism for effective coupling to the incident light field limits the potential of standalone non-plasmonic catalytic structures for photocatalysis. ${ }^{32,33}$

Optimal metal photocatalysts should combine the excellent light-harvesting properties of plasmonic nanostructures with the more versatile catalytic and photocatalytic properties of nonplasmonic, catalytic nanostructures. Approaches to achieve this multifunctionality have primarily relied on the synthesis of alloyed or directly interfaced nanostructures (e.g. Au/Pd), ${ }^{34-37}$ which modify the inherent behavior of each constituent, influencing both the local field enhancements and the catalytic characteristics. Other approaches have utilized top-down lithographic techniques, by which 2-dimensional films of photocatalysts were obtained. ${ }^{38-41}$ Recently it has been shown that Pd nanoparticle-decorated, oxide-coated Al nanocrystals enable efficient plasmon-induced photocatalysis at the Pd surface, where the plasmonic and catalytic functions were effectively distinct. ${ }^{42}$ This general structure has been called an "antenna-reactor" complex, where the plasmonic nanoparticle is the antenna and the catalytic particle, which can be interchanged based on desired reactivity, is the reactor. In an antenna-reactor complex, the local field induced by the photoexcited antenna particle drives a "forced" plasmon in the nonplasmonic, catalytic nanoparticle: this forced plasmon can serve as a direct source of hot carriers in the catalytic particle to drive chemical processes without the need for charge transfer between 
the antenna and the reactor components of the nanocomplex. In the initial demonstrations of antenna-reactor coupling, however, the optimal geometric configuration of the constituent materials to maximize photocatalytic performance was not addressed. Furthermore, while it is relatively straightforward to conclude that localizing a plasmonic particle near a non-plasmonic catalytic particle will enhance light absorption in the non-plasmonic particle, it is not clear how this translates to enhanced photocatalysis in the light-limited regime, where all photons must be utilized in a 3-D packed bed of catalytic particles. Optimizing photocatalytic complexes for highest efficiency photon management in the light-limited regime is of critical importance for further development of this approach.

In the work reported here, a series of antenna-reactor complexes consisting of core@shell/satellite $\left(\mathrm{Ag} @ \mathrm{SiO}_{2} / \mathrm{Pt}\right)$ heterostructures with varying Ag core diameter $(12,25,50$ and $100 \mathrm{~nm}$ ) were synthesized and their photocatalytic properties for the kinetically well-defined $\mathrm{CO}$ oxidation reaction were quantitatively compared. Rigorous quantum yield measurements in the light-limited regime demonstrate that for heterostructures with Ag nanoparticles (Ag NPs) in an intermediate size range of 25 and $50 \mathrm{~nm}$ diameter, plasmon-enhanced photocatalytic performance was observed at rates more than four times larger than for either smaller (12 nm diameter) or larger (100 nm diameter) Ag particles. Using optical and Monte Carlo simulations, it was shown that if the Ag NPs were too large, the heterostructures scattered light out of the catalyst bed, reducing photocatalytic reactivity. If the Ag NPs were too small, local field enhancements at the Pt-CO interface, where catalysis occurs, were reduced, and the Ag NPs would act as the primary optical absorbers. For optimal photocatalysis, the structures should be designed not only to maximize local field enhancements at the catalytic interface, but also to optimize photon penetration into the $3 \mathrm{D}$ ensemble of photocatalytic heterostructures in the 
catalyst bed, where each heterostructure behaves locally both as an absorber and a scatterer of the incident light.

To exploit the enhanced local fields of illuminated plasmonic nanoparticles to drive photocatalysis on non-plasmonic nanoparticles, the catalytically active nanoparticles (Pt NPs in this case) should be positioned within the near field of the plasmonic nanoparticle (Ag NPs in this case) to enable near-field coupling. ${ }^{43}$ The antenna-reactor complexes synthesized for this study were designed to exploit this type of coupling. They are concentric, core@shell/satellites heterostructures consisting of $\sim 5 \mathrm{~nm}$ diameter Pt NPs deposited on $\mathrm{SiO}_{2}$ coated spherical Ag NPs. A pinhole-free $\mathrm{SiO}_{2}$ spacer layer between the Ag NP surface and the Pt NPs was introduced to avoid potential catalytic activity from $\mathrm{Ag}$ itself, and to stabilize the heterostructure under reaction conditions. Although the $\mathrm{SiO}_{2}$ layer weakens the electromagnetic coupling between $\mathrm{Ag}$ and $\mathrm{Pt}$, this thin $(\sim 8-10 \mathrm{~nm})$ dielectric minimizes interfacial plasmon damping, which would otherwise occur at the Pt-Ag interface. It also increases the electromagnetic field energy density at the catalytic Pt surface. Both these effects are beneficial for enhancement of the local field of the Ag NP at the Pt surface.

The strategy used to synthesize the $\mathrm{Ag} @ \mathrm{SiO}_{2} / \mathrm{Pt}$ heterostructures is illustrated in Fig. 1A. Ag NPs were synthesized using a colloidal method where seed-mediated consecutive growth stages enabled precise control of Ag NP size $(12 \pm 2,25 \pm 3,51 \pm 5$ and $103 \pm 8 \mathrm{~nm}$ diameter Ag spheres were obtained, which in our discussion are labelled 12, 25, 50, and $100 \mathrm{~nm} \mathrm{Ag,} \mathrm{respectively).} \mathrm{Ag}$ NPs were coated with $\mathrm{SiO}_{2}$ using tetraethoxysilane (TEOS) hydrolysis, where the $\mathrm{SiO}_{2}$ thickness was controlled by the amount of TEOS added to the solution and kept constant at a uniform $\sim 8$ $10 \mathrm{~nm}$ thickness for all $\mathrm{Ag}$ sizes. Although a thinner $\mathrm{SiO}_{2}$ layer would be beneficial for electromagnetic coupling of the Pt catalytic particles to the Ag antenna particles, a spacer layer 
thickness of 8-10 nm was used to ensure constant layer thickness and formation of layers that were pinhole free.
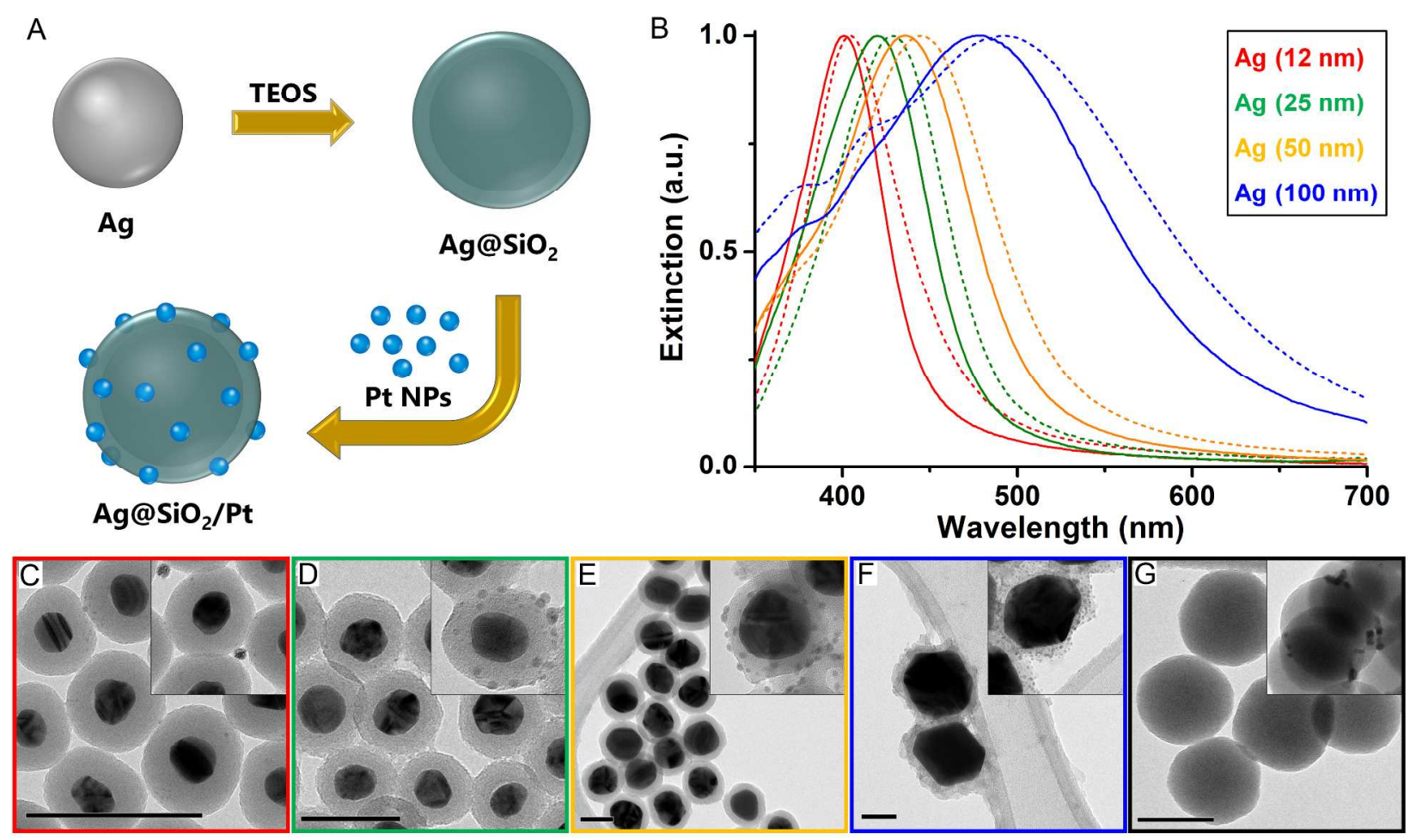

Figure 1. (A) Schematic of the synthesis strategy for the $\mathrm{Ag} @ \mathrm{SiO}_{2} / \mathrm{Pt}$ heterostructure for plasmon-enhanced photocatalysis on Pt. (B) UV-vis extinction spectra of the Ag (solid curves) and $\mathrm{Ag} @ \mathrm{SiO}_{2}$ (dash curves) structures with varying size $\mathrm{Ag}$ cores and constant $\sim 8-10 \mathrm{~nm} \mathrm{SiO}_{2}$ shells. (C-F) TEM images of the Ag@ $\mathrm{SiO}_{2}$ structures with varying size Ag cores and constant $\sim 8-10 \mathrm{~nm} \mathrm{\textrm {SiO } _ { 2 }}$ shells. Insets of $(\mathrm{C}-\mathrm{F})$ show corresponding $\mathrm{Ag} @ \mathrm{SiO}_{2} / \mathrm{Pt}$ heterostructures. The colors of curves in (B) and borders in $(\mathrm{C}-\mathrm{F})$ denote the size of Ag NPs: $12 \mathrm{~nm}(\mathrm{red}), 25 \mathrm{~nm}$ (green), $50 \mathrm{~nm}$ (yellow), and $100 \mathrm{~nm}$ (blue). (G) TEM images of the synthesized $\mathrm{SiO}_{2}$ spheres. Inset of $(\mathrm{G})$ shows corresponding $\mathrm{SiO}_{2} / \mathrm{Pt}$ heterostructures. All scale bars in $(\mathrm{C}-\mathrm{G})$ are $50 \mathrm{~nm}$.

The characteristic plasmon peak wavelength redshifted with increases in Ag size (Figure S1) and 
with the $\mathrm{SiO}_{2}$ coating, as expected (Figure 1B and Table S1). 5nm Pt NPs $(5.2 \pm 1.3 \mathrm{~nm}$ ) were synthesized separately from the $\mathrm{Ag} @ \mathrm{SiO}_{2}$ structures, where a Pt precursor $\left(\mathrm{H}_{2} \mathrm{PtCl}_{6}\right)$ was reduced by $\mathrm{NaBH}_{4}$, and sodium citrate was used as a capping agent, which was removed prior to catalysis by calcination. Then the as-synthesized Pt NPs were bound to the $\mathrm{Ag} @ \mathrm{SiO}_{2}$ structures through impregnation. Transmission Electron Microscopy (TEM) images of Ag@ $\mathrm{SiO}_{2}$ structures with varying $\mathrm{Ag}$ sizes and a constant $8-10 \mathrm{~nm} \mathrm{SiO}_{2}$ shell are shown in Figure 1C-F, and images of corresponding $\mathrm{Ag} @ \mathrm{SiO}_{2} / \mathrm{Pt}$ heterostructures are shown in insets. To interrogate differences between the photocatalytic performances of Pt NPs with and without antenna near-field coupling, we also prepared Pt NPs supported on bare $50 \mathrm{~nm}$ diameter $\mathrm{SiO}_{2}$ spheres synthesized via the Stöber method (Figure $1 \mathrm{G})$. The weight loading of Pt in all structures was identical (1.0\% with respect to the total weight of the heterostructures) so that the photocatalytic properties could be compared directly, without sample-to-sample variations. At constant Pt weight loading, the total number of Pt NPs per $\mathrm{Ag} @ \mathrm{SiO}_{2}$ structure is expected to increase with increasing $\mathrm{Ag}$ size, as is seen in the TEM images, however, the total number of Pt NPs loaded in the catalyst bed that perform photocatalysis was kept constant across all samples (see experimental details in Supporting Information). We note that there is some inhomogeneity in the $\mathrm{SiO}_{2}$ thickness on the $\mathrm{Ag}(100 \mathrm{~nm}) @ \mathrm{SiO}_{2} / \mathrm{Pt}$ heterostructure. Although we expect this not to influence comparison with the other structures due to catalytic measurements being the collective behavior over a huge number of particles.

We used $\mathrm{CO}$ oxidation by $\mathrm{O}_{2}$ as a model reaction to examine photocatalytic activity of the $\mathrm{Ag} @ \mathrm{SiO}_{2} / \mathrm{Pt}$ heterostructures. We have previously developed detailed insights into the mechanism of thermal and photocatalytic CO oxidation on Pt NPs, allowing us to focus here on how proximal Ag antennas influence Pt photocatalysis in the context of this reaction. ${ }^{31,44}$ The 
synthesized heterostructures were diluted to $1 / 5$ by weight with $\mathrm{SiO}_{2}$ gel (60 200 mesh), considered optically and chemically inert, to reduce the spatial density of the heterostructures and avoid catalytic mass and heat transfer limitations. Reactions were performed in a continuous flow isothermal packed bed reactor, which allowed for catalyst illumination and temperature control. ${ }^{14,15,31}$ Thermal kinetic measurements (performed without illumination) showed that the synthesized heterostructures exhibited essentially identical activation barriers and very similar (a maximum of 2-fold different) $\mathrm{CO}$ oxidation reaction rates per gram of $\mathrm{Pt}$, meaning that the $\mathrm{Pt}$ in all samples exhibited consistent inherent catalytic properties (Figure S2). In this reaction, kinetic measurements under identical conditions showed that $\mathrm{CO}$ desorption is the rate-limiting step (RLS), because the Pt surface is poisoned by a complete monolayer of $\mathrm{CO}$ under reaction conditions. ${ }^{45}$ Then, when the catalyst is illuminated under operating conditions, any increases in reaction rate $\left(\mathrm{CO}_{2}\right.$ production) are driven by increased rates of Pt-CO bond breaking, creating available Pt sites for $\mathrm{O}_{2}$ adsorption, dissociation, and $\mathrm{CO}_{2}$ formation. ${ }^{45}$

Photocatalysis was driven by continuous wave illumination from a Xenon lamp, which was used either in a broadband white light mode with a maximum photon flux of $\sim 600 \mathrm{~mW} / \mathrm{cm}^{2}$ (spectral output shown in Figure S3), or as spectrally filtered bands with $\sim 45 \mathrm{~nm}$ full-width at halfmaximum and a maximum photon flux of $\sim 120 \mathrm{~mW} / \mathrm{cm}^{2}$. We used illumination by wavelengths in the range of 350-700 nm, where direct photoexcitation of intramolecular HOMO-LUMO electronic transitions localized solely in $\mathrm{CO}$ is excluded and all photon absorption involves excitation of the metals. ${ }^{31}$ Photocatalytic rates (Eq. 1) were calculated as the difference of reaction rates (molecules/s) under illumination (photothermal) and in the dark (thermal), see Figure S4: 
Photocatalytic rate $\left(\frac{\text { molecules }}{s}\right)=$ Photothermal rate $\left(\frac{\text { molecules }}{s}\right)-$ Thermal rate $\left(\frac{\text { molecules }}{s}\right)$

Photoenhancements (Eq. 2) were defined as the ratio between the steady state photothermal reaction rate and the thermal reaction rate at the same temperature, see Figure S4:

$$
\text { Photoenhancement }=\frac{\text { Photothermal rate }\left(\frac{\text { molecules }}{s}\right)}{\text { Thermal rate }\left(\frac{\text { molecules }}{s}\right)}
$$

Quantum yields (QY, Eq. 3) were calculated by normalizing the photocatalytic rate (molecules/s) to the photon flux (photons/s).

$$
\text { Quantum Yield }\left(\frac{\text { molecules }}{\text { photon }}\right)=\frac{\text { photocatalytic rate }\left(\frac{\text { molecules }}{s}\right)}{\text { Photon flux }\left(\frac{\text { photons }}{s}\right)}
$$

In all photocatalytic measurements, similar background thermal rates were used by finely controlling the reaction temperature to ensure there was no influence of the magnitude of the thermal rate on the comparison of photocatalytic properties. ${ }^{15,31}$ The catalyst bed was thick enough that there was no light transmission through the catalyst bed, ensuring that the system was operated in the light-limited regime and justifying comparisons of the QY measurements (Figure S5). Performing photocatalytic reactions in the light-limited regime is critical for direct comparison of the influence of nanostructure geometry on reaction efficiency where the ultimate goal is to optimize performance on a per photon basis under conditions where all photons are used. The identical Pt weight loading and thermal catalytic properties of all heterostructures, combined with ensured measurements of photocatalysis in the light-limited regime at similar background thermal rates, enables a rigorous and quantitative comparison of photocatalytic QY on each sample with the only variable changes being the Ag optical properties due to nanoparticle size. 

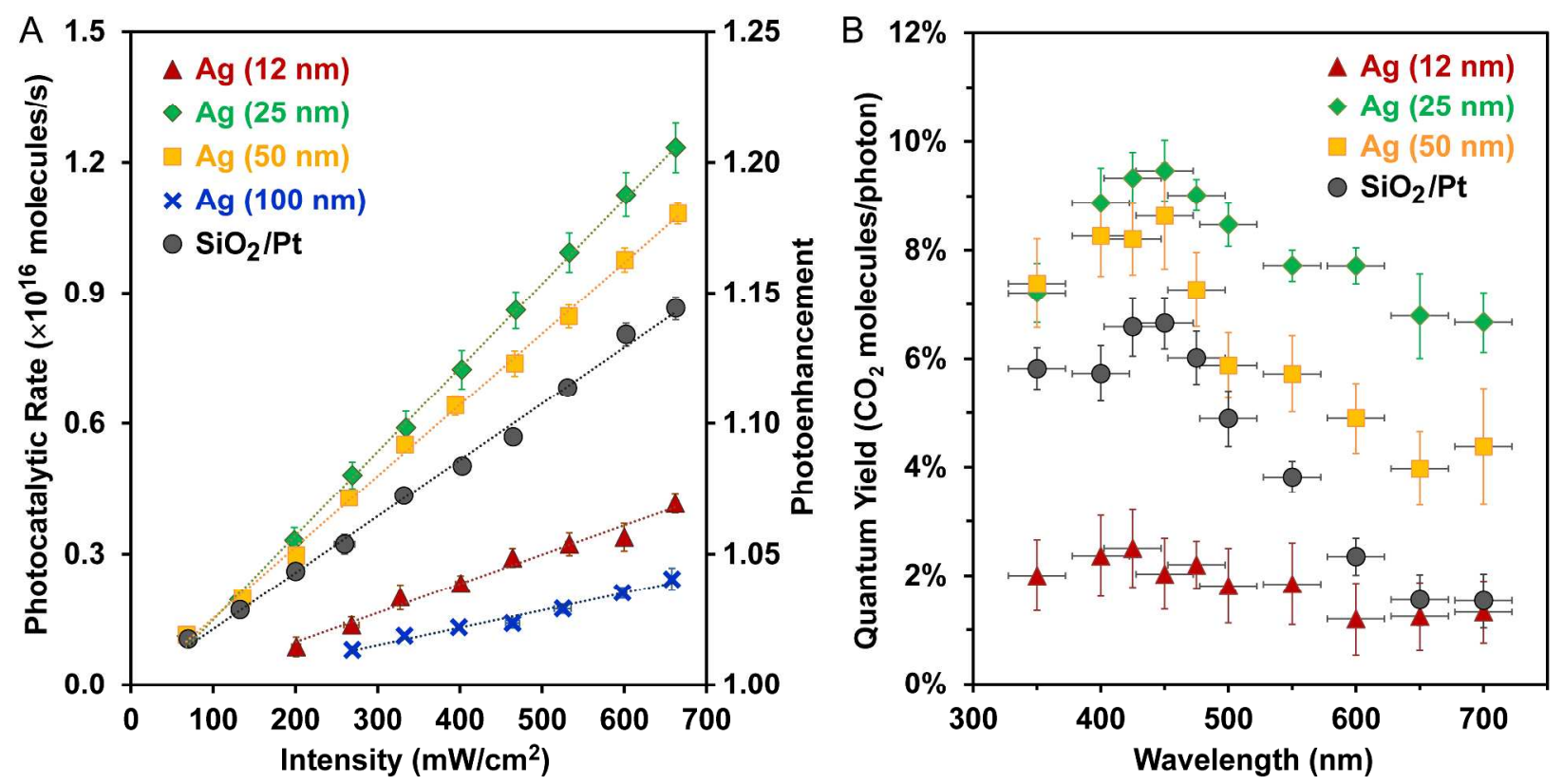

Figure 2. (A) Photocatalytic rate and photoenhancement of catalytic reactivity as a function of light intensity for the $\mathrm{Ag} @ \mathrm{SiO}_{2} / \mathrm{Pt}$ heterostructures with different Ag NP sizes. Results for Pt NPs deposited on $\mathrm{SiO}_{2}$ are also shown. Error bars associated with intensity ( $x$-direction) and photocatalytic rate and photoenhancement ( $y$-direction) are the standard error of three set of unique measurements. Broadband light illumination (Figure S3) was used for these experiments. (B) Quantum Yield (QY) as a function of light wavelength for $\mathrm{CO}$ oxidation on the $\mathrm{Ag} @ \mathrm{SiO}_{2} / \mathrm{Pt}$ heterostructures with different Ag NP sizes. Results for Pt NPs deposited on $\mathrm{SiO}_{2}$ are also shown. Error bars associated with wavelength ( $x$-direction) and QY ( $y$-direction) represent the full-width at half-maximum of the monochromator spectral output and the standard error of three unique measurements, respectively.

The light intensity-dependent photocatalytic rate and the photoenhancement of CO oxidation reactivity under broadband illumination for the five different heterostructures is shown in Figure 2A. The linear dependence of photocatalytic rate on photon flux provides evidence that photocatalysis is driven by single photon absorption events, and that heating (where an 
exponential dependence would be expected) is minimal in these systems. ${ }^{15,31}$ The linear dependence is consistent with the mechanism of photon-induced reactions driven by transient localization of excited charge carriers on adsorbates. ${ }^{14,46}$ Interestingly, we found the photocatalytic rate for $\operatorname{Ag}(25 \mathrm{~nm}) @ \mathrm{SiO}_{2} / \mathrm{Pt}$ was slightly higher than that for $\operatorname{Ag}(50$ $\mathrm{nm}) @ \mathrm{SiO}_{2} / \mathrm{Pt}$, while both were higher than that of $\mathrm{SiO}_{2} / \mathrm{Pt}$ (no Ag) catalysts. For example, the photocatalytic rate on $\mathrm{Ag}(25 \mathrm{~nm}) @ \mathrm{SiO}_{2} / \mathrm{Pt}$ was 1.49 -times greater than on $\mathrm{SiO}_{2} / \mathrm{Pt}$. However, the heterostructures with 12 or $100 \mathrm{~nm} \mathrm{Ag} \mathrm{only} \mathrm{weakly} \mathrm{enhanced} \mathrm{CO} \mathrm{oxidation} \mathrm{activity} \mathrm{under} \mathrm{white}$ light illumination, and in these cases the photocatalytic rates were actually lower than that of $\mathrm{SiO}_{2} / \mathrm{Pt}$. For example, the $\mathrm{Ag}(12 \mathrm{~nm}) @ \mathrm{SiO}_{2} / \mathrm{Pt}$ sample exhibited 0.44-times the photocatalytic rates measured on $\mathrm{SiO}_{2} / \mathrm{Pt}$.

Wavelength-dependent QYs for photocatalytic CO oxidation over Pt catalysts deposited on $\mathrm{Ag} @ \mathrm{SiO}_{2}$ or $\mathrm{SiO}_{2}$ structures are shown in Figure 2B. The low photocatalytic rates for $\mathrm{Ag}(100$ nm)@ $\mathrm{SiO}_{2} / \mathrm{Pt}$ made the wavelength dependence difficult to resolve, so these results were omitted. The $\mathrm{CO}$ oxidation QYs measured for $\mathrm{SiO}_{2} / \mathrm{Pt}$ (no $\mathrm{Ag}$ ) exhibited a resonant deviation from the absorption spectrum of $\mathrm{SiO}_{2} / \mathrm{Pt}$ catalysts between $375-600 \mathrm{~nm}$, with a peak QY at $\sim 450$ nm. These results (both the resonant behavior and magnitude of QY) are in quantitative agreement with our previous study, ${ }^{31}$ which demonstrated that direct photoexcitation of hybridized $\mathrm{Pt}-\mathrm{CO}$ states was the dominant mechanism driving photocatalysis on the Pt NPs. Rather than photons being absorbed by $\mathrm{Pt}$, followed by hot carrier transient transfer to $\mathrm{CO}$ being responsible for driving $\mathrm{CO}$ desorption, photocatalysis in this system is mediated by direct absorption of photons by electronic transitions localized at the Pt-CO interface. The excitation process induces $\mathrm{CO}$ desorption by non-adiabatic vibrational energy deposition, which creates vacant Pt sites for the catalytic cycle. At each wavelength, QYs for $\mathrm{Ag}(25 \mathrm{~nm}) @ \mathrm{SiO}_{2} / \mathrm{Pt}$ are 
slightly higher than those for $\mathrm{Ag}(50 \mathrm{~nm}) @ \mathrm{SiO}_{2} / \mathrm{Pt}$, and in general the relative magnitudes of the QYs measured from all catalysts are in excellent agreement with the broadband intensitydependent measurements.

From our quantitative photocatalytic measurements, we find that both the QY and the wavelength dependence of photocatalytic $\mathrm{CO}$ oxidation on $\mathrm{Ag} @ \mathrm{SiO}_{2} / \mathrm{Pt}$ heterostructures are influenced by Ag NP size in a complex manner. The magnitude of the measured photocatalytic rates and QYs are not proportional to the size of the Ag core, but rather, there is an optimal Ag NP size that enhances photocatalysis. This is somewhat surprising, since localizing catalytic metals in the near field of plasmonic particles is generally believed to enhance photocatalytic properties. ${ }^{34-42}$ However, few of these measurements were conducted in a light-limited regime. In this regime, Ag NP size influences the optical properties of the heterostructures through two mechanisms: the branching ratio of radiative and non-radiative plasmon decay, and the modulation of the magnitude of local electromagnetic field enhancements at the catalytic Pt surface. 


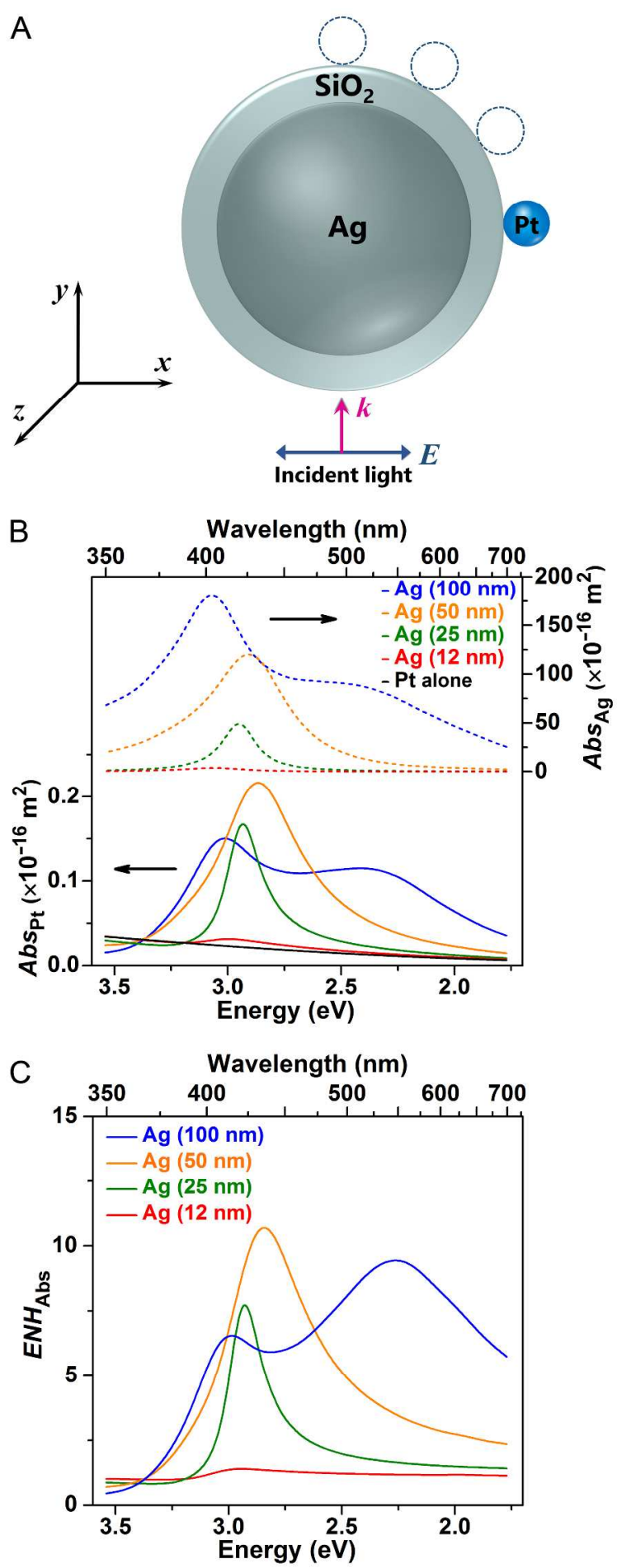

Figure 3. (A) Schematic of the general model structure used for FDTD simulations in this study. Blue dash circles show the other three considered Pt NP positions. (B) Absorption cross section in $\mathrm{Pt}\left(A b s_{\mathrm{Pt}}\right.$, solid curves, left axis) and $\mathrm{Ag}$ ( $A b s_{\mathrm{Ag}}$, dash curves, right axis), and (C) Pt absorption enhancement $\left(E N H_{\mathrm{Abs}}\right)$ as a function of wavelength for $\mathrm{Ag} @ \mathrm{SiO}_{2} / \mathrm{Pt}$ heterostructures with 
different Ag NP sizes and a constant $10 \mathrm{~nm} \mathrm{SiO}_{2}$ shell.

To theoretically analyze how $\mathrm{Ag} \mathrm{NP}$ size influenced light absorption in $\mathrm{Ag} @ \mathrm{SiO}_{2} / \mathrm{Pt}$ heterostructures, finite-difference time-domain (FDTD) simulations were performed. Ag NPs with varying sizes $(12,25,50$, and $100 \mathrm{~nm})$ were employed as the plasmonic cores, which were coated by a constant $10 \mathrm{~nm} \mathrm{SiO}_{2}$ shell and decorated with one $5 \mathrm{~nm}$ Pt NP. Due to the polarized and directional light flux used in our FDTD simulations, the position of the Pt NPs on the $\mathrm{SiO}_{2-}$ coated Ag NP can significantly influence coupling between the antenna NP and the catalytic NP. However, since our experiments utilized unpolarized light and randomly oriented heterostructures, we approximate our experimental systems by placing Pt NPs in the top-right quarter at the $x-y$ plane of the $\mathrm{Ag} @ \mathrm{SiO}_{2}$ structure at four locations from 0 to $90^{\circ}$ (Figure $3 \mathrm{~A}$ ) and averaging the response for this range of orientations. We propose further justified assumptions to facilitate comparison of the theoretical and experimental systems (Section 4.1 and Figure S6-8, Supporting Information).

While our simulations of the effect of the $\mathrm{SiO}_{2}$ shell thickness clearly demonstrated that a thinner $\mathrm{SiO}_{2}$ shell is highly advantageous for facilitating Pt coupling to the Ag NP plasmon (Section 4.3 and Figure S9-11, Supporting Information), we here use $10 \mathrm{~nm}$-thickness $\mathrm{SiO}_{2}$ layer and focus on the influence of Ag NP size on catalytic efficiency. The position-averaged light absorption cross sections in $\mathrm{Pt}$ and $\mathrm{Ag}$ in the $\mathrm{Ag} @ \mathrm{SiO}_{2} / \mathrm{Pt}$ heterostructures, and in an isolated Pt NP for comparison, are shown in Figure 3B (see further details in Figure S12). For each structure, the spectral shapes of the absorption cross sections in Pt were similar to the spectral shapes of absorption cross sections in Ag, indicating the enhancement of Pt absorption occurred through near-field coupling. The calculated absorption cross section in $\mathrm{Pt}$ in the $\mathrm{Ag} @ \mathrm{SiO}_{2} / \mathrm{Pt}$ 
heterostructures are significantly enhanced from that of the isolated Pt NP. In contrast, there is no notable enhancement for the absorption cross section in $\mathrm{Pt}$ for $\mathrm{SiO}_{2}(50 \mathrm{~nm}) / \mathrm{Pt}$ structures (Figure S13). Light absorption by $\mathrm{Pt}$ in the $\mathrm{Ag} @ \mathrm{SiO}_{2} / \mathrm{Pt}$ heterostructures shows a strong dependence on Ag NP size. From Figure 3B, it is seen that for the $12 \mathrm{~nm} \mathrm{Ag} \mathrm{NP} \mathrm{(red),} \mathrm{the} \mathrm{cross} \mathrm{section} \mathrm{for} \mathrm{light}$ absorption by $\mathrm{Pt}$ is small, closer to that of the isolated Pt NP case (black). For the two intermediate-sized Ag NP cases (green and yellow), the Pt absorption lies in the 400-450 nm wavelength spectral window and is significantly enhanced compared to the isolated Pt case. For the $100 \mathrm{~nm} \mathrm{Ag} \mathrm{NPs} \mathrm{(blue),} \mathrm{the} \mathrm{Pt} \mathrm{absorption} \mathrm{is} \mathrm{strongly} \mathrm{redshifted} \mathrm{to} \sim 520 \mathrm{~nm}$, and the absorption in the spectral window of interest corresponds to the Ag NP quadrupole mode.

To quantitatively compare the influence of the Ag NP on Pt photon absorption, we defined the Pt absorption enhancement $\left(E N H_{\mathrm{Abs}}\right.$, Eq. 4), which is the ratio between the Pt absorption cross section enhanced by the Ag NP plasmon in the heterostructures and the absorption cross section of an isolated Pt NP alone.

$$
E N H_{\mathrm{Abs}}=\sum_{1}^{4} \frac{1}{4} \int \frac{A b s_{\mathrm{Pt}^{\# n}(E)}}{A b s_{\mathrm{Pt}^{*}(E)}} d E
$$

where $A b s_{\mathrm{Pt}}{ }^{*}$ is the absorption in an isolated $\mathrm{Pt} \mathrm{NP}$ without the $\mathrm{Ag} @ \mathrm{SiO}_{2}$ structure, $A b s_{\mathrm{Pt}}{ }^{\# n}$ is the absorption in $\mathrm{Pt}$ for $\mathrm{Ag} @ \mathrm{SiO}_{2} / \mathrm{Pt}$ with one Pt NP at $\# n$ position, and $\# n=1,2,3$, or 4 represents $0^{\circ}, 30^{\circ}, 60^{\circ}$, or $90^{\circ}$ position at the $x-y$ plane, respectively, see Figure $3 \mathrm{~A}$.

Figure $3 \mathrm{C}$ shows $E N H_{\mathrm{Abs}}$ for the various heterostructures. It is seen that the $E N H_{\mathrm{Abs}}$ exhibits similar magnitudes, although with different spectral shapes, for the heterostructures containing 25, 50 and $100 \mathrm{~nm} \mathrm{Ag} \mathrm{particles} \mathrm{and} \mathrm{is} \mathrm{significantly} \mathrm{diminished} \mathrm{for} \mathrm{the} 12 \mathrm{~nm} \mathrm{Ag} \mathrm{particle}$ heterostructure. If photocatalysis is conducted on 2-D films of antenna-reactor complexes, where 
all structures are illuminated with equal photon flux and the absorption efficiency by the film is much less than $100 \%$, then $E N H_{\text {Abs }}$ provides a complete description of how significantly the Ag NP plasmonic near field will enhance Pt light absorption (Figure S15). ${ }^{38-41}$ However, in a 3-D catalyst bed operated in the light-limited regime, it is not enough to simply consider how much Ag enhances Pt light absorption in discrete heterostructures, and thus the relative magnitude of $E N H_{\mathrm{Abs}}$ as a function of $\mathrm{Ag}$ particle size do not match the experimentally measured trends in photocatalytic rates (Figure 2).

In 3-D reactor volumes operating in the light-limited regime, the fate of all incident photons must be considered, as any photons not contributing directly to the photocatalytic process will reduce the measured photocatalytic reactivity. The Ag NP plasmon can decay through non-radiative and radiative channels, ${ }^{6,22,47}$ each of which may influence the overall photocatalytic performance of the heterostructures. Non-radiative plasmon decay and hot carrier generation cannot contribute to hot-carrier mediated photocatalysis by $\mathrm{Pt}$ because of the insulating pinhole free $\mathrm{SiO}_{2}$ layer separating Ag and Pt. Although photon absorption by Ag could contribute indirectly to photocatalysis by Pt through heating of the heterostructure, the experimentally observed linear dependent of rate on light intensity suggests that heating is minimal at the experimentally explored light intensities. Plasmonic nanoparticles with diameters $>50 \mathrm{~nm}$ are efficient antennas that can re-radiate photons out of the catalyst bed, eliminating any possibility of contributing to photocatalysts. In an attempt to address these various effects, we defined performance metrics to evaluate plasmon-enhanced Pt NPs absorption in the examined heterostructures that account for absorption of photons by Ag and scattering of photons by Ag without Pt absorption (Section 4.4, Supporting Information). ${ }^{48}$ However, it is seen that none of the performance metrics defined based on the FDTD simulations of single heterostructures could capture the trends in 
photocatalytic performance observed in Figure 2. Thus, we conclude that the collective behavior of the heterostructures in the 3-D catalyst bed must be considered simultaneously to account for the photocatalytic performance.

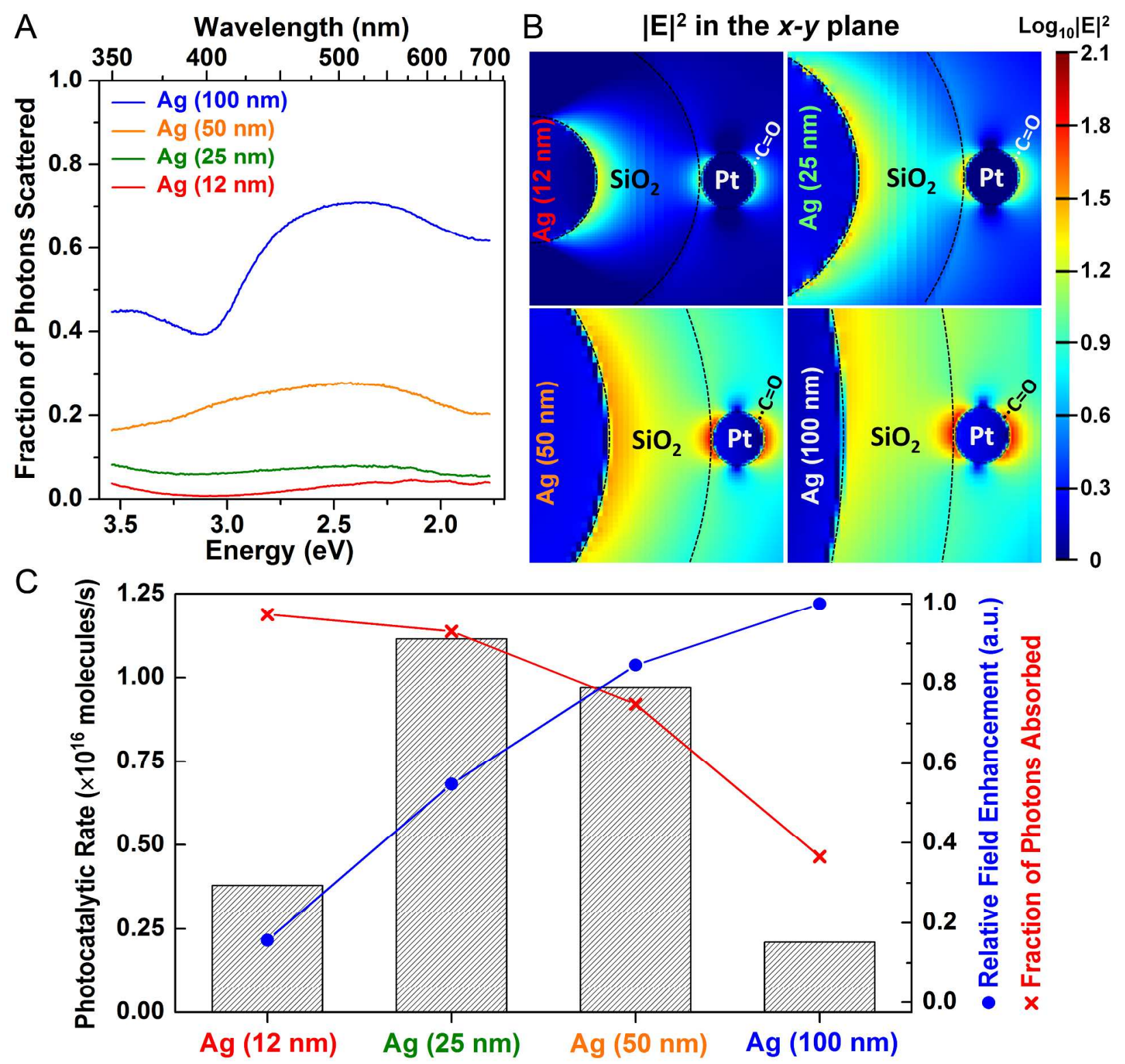

Figure 4. (A) Wavelength-dependent fraction of photon scattered out of the catalyst bed for $\mathrm{Ag} @ \mathrm{SiO}_{2} / \mathrm{Pt}$ heterostructures with different Ag NP sizes derived from Monte Carlo simulations. (B) Electromagnetic field distributions at Pt surfaces in the $x-y$ plane at $500 \mathrm{~nm}$ for the four 
$\mathrm{Ag} @ \mathrm{SiO}_{2} / \mathrm{Pt}$ heterostructures with different Ag NP sizes. FDTD simulations were conducted with the model shown in Figure 3A. (C) Measured photocatalytic rates under $600 \mathrm{~mW} / \mathrm{cm}^{2}$ white light (column bars), relative field enhancements at Pt surface from FDTD calculations (blue dots), and fractions of photons absorbed in the catalyst bed from Monte Carlo simulations (red crosses) for the four $\mathrm{Ag} @ \mathrm{SiO}_{2} / \mathrm{Pt}$ heterostructures with different Ag NP sizes. The relative field enhancements and fraction of photons absorbed in the catalyst bed were calculated based on Pt position averaging and the broadband illumination shown in Figure S3.

To quantitatively probe the fate of photons in the examined 3-D catalyst beds, a Monte Carlo simulation approach was used to model light transport in catalyst beds consisting of $\mathrm{Ag} @ \mathrm{SiO}_{2} / \mathrm{Pt}$ heterostructures dispersed in $\mathrm{SiO}_{2}$ diluents (Section 5, Supplemental Information). ${ }^{49,50}$ The experimental $\mathrm{SiO}_{2} /$ photocatalyst volumetric dilution ratio, which defined the particles density in the catalyst bed (Table S3), and FDTD calculated optical properties of the $\mathrm{Ag} @ \mathrm{SiO}_{2} / \mathrm{Pt}$ heterostructures were used to simulate each system. These simulations provide a quantitative prediction of the distribution of photons in the 3-D catalyst bed, including their average penetration depth, where they are primarily absorbed, and whether they are simply reflected out of the catalyst bed. The average penetration depth of photons in a catalyst bed loaded with each type of $\mathrm{Ag} @ \mathrm{SiO}_{2} / \mathrm{Pt}$ heterostructure was calculated as a function of photon wavelength, showing that photons at all wavelengths (except within $\sim 20 \mathrm{~nm}$ of the LSPR peak) penetrate deeper into catalyst beds that are packed with smaller Ag NPs (Figure S16).

To interpret our experimental results, the fraction of total incident photons that were scattered out of the catalyst bed was calculated as a function of wavelength (Figure 4A). For the case of $\mathrm{Ag}(100 \mathrm{~nm}) @ \mathrm{SiO}_{2} / \mathrm{Pt}$ heterostructures, 40-70\% (depending on wavelength) of all photons were scattered out of the catalyst bed and inaccessible for photocatalysis. The fraction of total 
photons scattered from the catalyst bed significantly decreases with decreasing Ag core size, where for the $\mathrm{Ag}(12 \mathrm{~nm}) @ \mathrm{SiO}_{2} / \mathrm{Pt}$ case $<5 \%$ of photons were scattered from the catalyst bed at all wavelengths, consistent with the size-dependent branching ratio of radiative and non-radiative NP plasmon decay. The Monte Carlo simulations provide excellent insight into why $\operatorname{Ag}(100$ $\mathrm{nm}) @ \mathrm{SiO}_{2} / \mathrm{Pt}$ heterostructures performed poorly as photocatalysts. This is because the strong radiative behavior of the heterostructures scatters $\sim 40-70 \%$ of the incoming photons out of the catalyst bed. While these simulations provide a clear mechanistic explanation for the poor performance of $\mathrm{Ag}(100 \mathrm{~nm}) @ \mathrm{SiO}_{2} / \mathrm{Pt}$ heterostructures, they still cannot explain why the photocatalytic performance decreased with decreasing Ag size from 25 to $12 \mathrm{~nm}$, a regime of high optical absorption and weak light scattering.

To understand why $\mathrm{Ag} @ \mathrm{SiO}_{2} / \mathrm{Pt}$ heterostructures with 25 and 50 nm Ag cores were optimal for photocatalysis, we consider that photocatalytic $\mathrm{CO}$ oxidation on $\mathrm{Pt}$ is not simply driven by light absorption in $\mathrm{Pt}$, but instead by the Ag antenna induced near-field enhancement at the Pt-CO interface that can induce a direct interfacial electronic transition. This was demonstrated by the resonant wavelength dependence of $\mathrm{QY}$ for $\mathrm{Pt} / \mathrm{SiO}_{2}$ (Figure 2B). Because of this, the electromagnetic field strength at the Pt-CO interface, rather than light absorption by the Pt NP, controls photocatalysis. In this mechanism, the enhanced near-fields at the Pt-CO interface due to localization near Ag increases the rate of direct photoexctiation of the Pt-CO bond and thus the rate of $\mathrm{CO}$ oxidation. We simulated the electromagnetic field density distribution at the $\mathrm{Pt}$ surfaces (see Section 6, Supplemental Information) as a function of incident light wavelength for each heterostructure. Generally, larger $\mathrm{Ag}$ cores result in stronger electromagnetic field enhancement at Pt surfaces (Figure S17-18). This can be visually seen in Figure 4B, where the electromagnetic field distributions at the Pt surfaces on each heterostructure under $500 \mathrm{~nm}$ 
wavelength light illumination are shown. It is clear that the field intensity at the Pt-CO interface increases with increasing $\mathrm{Ag}$ size. Thus, the optimum photocatalytic performance of $\operatorname{Ag}(25$ or 50 $\mathrm{nm}) @ \mathrm{SiO}_{2} / \mathrm{Pt}$ occurs because these structures provide the most effective balance of photons absorbed within the catalyst bed (not scattered from the top) while still significantly enhancing the field intensity at the Pt-CO interface.

To directly show this balance, the photocatalytic rates under broadband light illumination (measured at $600 \mathrm{~mW} / \mathrm{cm}^{2}$, although the trend is similar at all photon fluxes) were plotted simultaneously with the field enhancements at the Pt surface (simulated by FDTD method) and the photon fraction absorbed in the catalyst bed (calculated by Monte Carlo approach) for each heterostructure with varying Ag NP size (Figure 4C). The field enhancements and absorbed photon fractions were calculated as weighted averages with respect to the broadband spectrum of the light source in our photocatalysis measurements followed by normalization, to allow comparison with photocatalysis measurements conducted with a broadband photon flux. As the Ag NP size is increased, the relative field enhancement at the Pt-CO interface increases, but the fraction of total photons absorbed by the catalyst bed decreases. Thus, we find that when Ag particles are large enough $(100 \mathrm{~nm})$ to exhibit a significant scattering cross section, photons are scattered out of the catalyst bed, ultimately reducing the photocatalytic performance of the $\mathrm{Ag} @ \mathrm{SiO}_{2} / \mathrm{Pt}$ heterostructures relative to the isolated $\mathrm{SiO}_{2} / \mathrm{Pt}$ structures. However, when the $\mathrm{Ag}$ NPs are small $(12 \mathrm{~nm})$, the local field enhancement at the catalytic Pt interface is minimal, with the small Ag NPs absorbing a large fraction of the incoming photons (Figure 3D), reducing the photocatalytic performance relative to the isolated $\mathrm{SiO}_{2} / \mathrm{Pt}$ structures. When $\mathrm{Ag}$ exists in an intermediate size $(25$ or $50 \mathrm{~nm}$ ), the incident photons penetrate deeply into the catalyst bed and generate intense local fields at the Pt surface, enabling the Ag NP antenna to effectively enhance 
the Pt catalytic performance compared to the isolated $\mathrm{SiO}_{2} / \mathrm{Pt}$ structures, even in the light-limited regime where all incident photons are used. Given the demonstrated importance of near-field enhancement magnitude at the Pt-CO interface, we further suggest that synthetic methods that reduce the thickness or dielectric function of the spacing layer between the plasmonic and catalytic nanoparticles will also be of critical importance for optimization of photocatalytic performance.

In summary, we have studied how the size dependence of the plasmonic antenna component of $\mathrm{Ag} @ \mathrm{SiO}_{2} / \mathrm{Pt}$ antenna-reactor photocatalysts can control photocatalytic $\mathrm{CO}$ oxidation activity at the Pt surface. In our experimental studies and theoretical analysis of this system, we examined how the relative roles of the plasmonic near-field enhancement, optical absorption, and multiple light scattering events in a concentrated 3-D catalyst bed geometry all influence the efficiency of these photocatalysts. This study shows that under the light-limited regime, an optimal plasmonic antenna size is required to enable enhanced plasmonic photocatalysis in an antenna-reactor complex. These results are expected to further stimulate the design of unique, multicomponent plasmonic photocatalyst heterostructures with optimized light-activation efficiencies, as well as to address the critical importance of photocatalyst concentration in 3-D catalyst beds.

\author{
ASSOCIATED CONTENT \\ The Supporting Information is available free of charge on the ACS Publications website. \\ Materials and methods, additional characterizations on $\mathrm{Ag} @ \mathrm{SiO}_{2} / \mathrm{Pt}$, prerequisites for \\ comparison on photocatalytic performance, supplementary discussions on FDTD simulations,


supplementary discussions on Monte Carlo simulations, and supplementary discussions on electromagnetic field enhancements.

\section{AUTHOR INFORMATION}

\section{Corresponding Author}

*E-mail: christopher@engr.ucr.edu.

\section{Notes}

The authors declare no competing financial interest.

\section{ACKNOWLEDGMENTS}

P.C. acknowledges partial funding for this work from the University of California, Riverside, Center for Catalysis, Army Research Office grant W911NF-15-1-0533, the Air Force Office of Scientific Research MURI Grant FA9550-15-10022, and the Welch Foundation grants C-1220 (NJH) and C-1222 (PN). TEM was carried out at UCR Central Facility for Advanced Microscopy and Microanalysis (CFAMM).

\section{REFERENCES}

(1) Moniz, S. J.; Shevlin, S. A.; Martin, D. J.; Guo, Z.-X.; Tang, J. Energy \& Envrion. Sci. 2015, 8, 731-759.

(2) Daghrir, R.; Drogui, P.; Robert, D. Ind. Eng. Chem. Res. 2013, 52, 3581-3599.

(3) Schultz, D. M.; Yoon, T. P. Science 2014, 343, 1239176.

(4) Hisatomi, T.; Kubota, J.; Domen, K. Chem. Soc. Rev. 2014, 43, 7520-7535.

(5) Rajeshwar, K.; Thomas, A.; Janáky, C. J. Phys. Chem. Lett. 2015, 6, 139-147.

(6) Cushing, S. K.; Wu, N. J. Phys. Chem. Lett. 2016, 7, 666-675.

(7) Peiris, S.; McMurtrie, J.; Zhu, H.-Y. Catal. Sci. Technol. 2016, 6, 320-338.

(8) Kale, M. J.; Christopher, P. Science 2015, 349, 587-588.

(9) Sakamoto, H.; Ohara, T.; Yasumoto, N.; Shiraishi, Y.; Ichikawa, S.; Tanaka, S.; Hirai, T. J. Am. Chem. Soc. 2015, 137, 9324-9332.

(10) Ross, M. B.; Mirkin, C. A.; Schatz, G. C. J. Phys. Chem. C 2016, 120, 816-830. 
(11) Zhu, H.; Ke, X.; Yang, X.; Sarina, S.; Liu, H. Angew. Chem., Int. Ed. 2010, 49, 9657-9661.

(12) Mukherjee, S.; Libisch, F.; Large, N.; Neumann, O.; Brown, L. V.; Cheng, J.; Lassiter, J. B.; Carter, E. A.; Nordlander, P.; Halas, N. J. Nano Lett. 2013, 13, 240-247.

(13) Mukherjee, S.; Zhou, L.; Goodman, A. M.; Large, N.; Ayala-Orozco, C.; Zhang, Y.; Nordlander, P.; Halas, N. J. J. Am. Chem. Soc. 2014, 136, 64-67.

(14) Christopher, P.; Xin, H.; Linic, S. Nat. Chem. 2011, 3, 467-472.

(15) Christopher, P.; Xin, H.; Marimuthu, A.; Linic, S. Nat. Mater. 2012, 11, 1044-1050.

(16) Boerigter, C.; Campana, R.; Morabito, M.; Linic, S. Nat. Commun. 2016, 7, 10545.

(17) Marimuthu, A.; Zhang, J.; Linic, S. Science 2013, 339, 1590-1593.

(18) Zhou, L.; Zhang, C.; McClain, M. J.; Manjavacas, A.; Krauter, C. M.; Tian, S.; Berg, F.; Everitt, H. O.; Carter, E. A.; Nordlander, P.; Halas, N. J. Nano Lett. 2016, 16, 1478-1484.

(19) Linic, S.; Christopher, P.; Ingram, D. B. Nat. Mater. 2011, 10, 911-921.

(20) Linic, S.; Christopher, P.; Xin, H.; Marimuthu, A. Acc. Chem. Res. 2013, 46, 1890-1899.

(21) Clavero, C. Nat. Photonics 2014, 8, 95-103.

(22) Kale, M. J.; Avanesian, T.; Christopher, P. ACS Catal. 2014, 4, 116-128.

(23) Linic, S.; Aslam, U.; Boerigter, C.; Morabito, M. Nat. Mater. 2015, 14, 567-576.

(24) Brongersma, M. L.; Halas, N. J.; Nordlander, P. Nat. Nanotechnol. 2015, 10, 25-34.

(25) Avanesian, T.; Christopher, P. J. Phys. Chem. C 2014, 118, 28017-28031.

(26) Govorov, A. O.; Zhang, H.; Gun'ko, Y. K. J. Phys. Chem. C 2013, 117, 16616-16631.

(27) Sarina, S.; Zhu, H. Y.; Xiao, Q.; Jaatinen, E.; Jia, J.; Huang, Y.; Zheng, Z.; Wu, H. Angew. Chem., Int. Ed. 2014, 53, 2935-2940.

(28) Wu, B.; Lee, J.; Mubeen, S.; Jun, Y. S.; Stucky, G. D.; Moskovits, M. Adv. Opt. Mater. 2016, 4, 1041-1046.

(29) Zhang, N.; Han, C.; Xu, Y.-J.; Foley Iv, J. J.; Zhang, D.; Codrington, J.; Gray, S. K.; Sun, Y. Nat. Photonics 2016, 10, 473-482.

(30) Ro, I.; Sener, C.; Stadelman, T. M.; Ball, M. R.; Venegas, J. M.; Burt, S. P.; Hermans, I.; Dumesic, J. A.; Huber, G. W. J. Catal. 2016, 344, 784-794.

(31) Kale, M. J.; Avanesian, T.; Xin, H.; Yan, J.; Christopher, P. Nano Lett. 2014, 14, 5405-5412.

(32) González-Díaz, J. B.; García-Martín, A.; Armelles, G.; Navas, D.; Vázquez, M.; Nielsch, K.; Wehrspohn, R. B.; Gösele, U. Adv. Mater. 2007, 19, 2643-2647.

(33) Zoric, I.; Zach, M.; Kasemo, B.; Langhammer, C. ACS Nano 2011, 5, 2535-2546.

(34) Huang, X.; Li, Y.; Chen, Y.; Zhou, H.; Duan, X.; Huang, Y. Angew. Chem., Int. Ed. 2013, 52, 6063-6067.

(35) Sarina, S.; Zhu, H.; Jaatinen, E.; Xiao, Q.; Liu, H.; Jia, J.; Chen, C.; Zhao, J. J. Am. Chem. Soc. 2013, 135, 5793-5801.

(36) Xiao, Q.; Sarina, S.; Jaatinen, E.; Jia, J.; Arnold, D. P.; Liu, H.; Zhu, H. Green Chem. 2014, 16, $4272-4285$.

(37) Zheng, Z.; Tachikawa, T.; Majima, T. J. Am. Chem. Soc. 2015, 137, 948-957.

(38) Larsson, E. M.; Langhammer, C.; Zorić, I.; Kasemo, B. Science 2009, 326, 1091-1094.

(39) Liu, N.; Tang, M. L.; Hentschel, M.; Giessen, H.; Alivisatos, A. P. Nat. Mater. 2011, 10, 631-636.

(40) Antosiewicz, T. J.; Wadell, C.; Langhammer, C. Adv. Opt. Mater. 2015, 3, 1591-1599.

(41) Zhang, C.; Zhao, H.; Zhou, L.; Schlather, A. E.; Dong, L.; McClain, M. J.; Swearer, D. F.; Nordlander, P.; Halas, N. J. Nano Lett. 2016, 16, 6677-6682.

(42) Swearer, D. F.; Zhao, H.; Zhou, L.; Zhang, C.; Robatjazi, H.; Martirez, J. M. P.; Krauter, C. M.; Yazdi, S.; McClain, M. J.; Ringe, E.; Carter, E. A.; Nordlander, P.; Halas, N. J. Proc. Natl. Acad. Sci. U. S. A. 2016, 113, 8916-8920.

(43) Ingram, D. B.; Christopher, P.; Bauer, J. L.; Linic, S. ACS Catal. 2011, 1, 1441-1447.

(44) Kale, M. J.; Christopher, P. ACS Catal. 2016, 6, 5599-5609.

(45) Allian, A. D.; Takanabe, K.; Fujdala, K. L.; Hao, X.; Truex, T. J.; Cai, J.; Buda, C.; Neurock, M.; Iglesia, E. J. Am. Chem. Soc. 2011, 133, 4498-4517.

(46) So, S.; Franchy, R.; Ho, W. J. Chem. Phys. 1991, 95, 1385-1399.

24 
1

2

3

4

5

6

7

8

9

10

11

12

13

14

15

16

17

18

19

20

21

22

23

24

25

26

27

28

29

30

31

32

33

34

35

36

37

38

39

40

41

42

43

44

45

46

47

48

49

50

51

52

53

54

55

56

57

58

59

60

(47) Evanoff, D. D.; Chumanov, G. J. Phys. Chem. B 2004, 108, 13957-13962.

(48) Khorashad, L. K.; Besteiro, L. V.; Wang, Z.; Valentine, J.; Govorov, A. O. J. Phys. Chem. C 2016, $13215-13226$.

(49) Hogan, N. J.; Urban, A. S.; Ayala-Orozco, C.; Pimpinelli, A.; Nordlander, P.; Halas, N. J. Nano Lett. 2014, 14, 4640-4645.

(50) Tanzid, M.; Hogan, N. J.; Sobhani, A.; Robatjazi, H.; Pediredla, A. K.; Samaniego, A.; Veeraraghavan, A.; Halas, N. J. ACS Photonics 2016, 3, 1787-1793. 